\title{
Transabdominal and Laparoscopic Myomectomy Comparative Analysis of 566 Cases
}

\author{
Minhua Gao*
}

\author{
Changzhou Maternity and Chidren Health Hospital, Changzhou, China
}

\begin{abstract}
The purpose of our study here is introducing curative effect by comparing and analyzing abdominal type myomectomy and laparoscopic myomectomy. We have compared and analyzed 301 cases of abdominal type myomectomy and 265 cases of laparoscopic myomectomy which were in our hospital from January 2003 to December 2008. The study shows that the time of abdominal type myomectomy is $87.18 \pm 36.80$ minutes, and the time of laparoscopic myomectomy is $81.56 \pm 30.71$ minutes. There isn't obvious difference between these two kinds of surgeries. The hemorrhagic volume in abdominal type myomectomy is $106.86 \pm 32.65 \mathrm{ml}$ and the hemorrhagic volume in laparoscopic myomectomy is $85.23 \pm 30.37 \mathrm{ml}$. The hemorrhagic volume difference in these two kinds of surgeries is very important from the point of view of statistics $(\mathrm{P}<0.01)$. The average days which used to restore in abdominal type myomectomy is $2.85 \pm 1.73$, and the average days which used to restore in laparoscopic myomectomy is $5.81 \pm 1.47$. And the difference is also very important $(\mathrm{P}<0.01)$. We have concluded that the laparoscopic myomectomy has such advantages as small hurt, rapid restoration and no incision. On the other hand, the hemorrhagic volume is very low and the restoration-days are very low.
\end{abstract}

Index Terms: Transabdominal; Laparoscopic; Myomectomy

(C) 2011 Published by MECS Publisher. Selection and/or peer review under responsibility of the Research Association of Modern Education and Computer Science.

\section{Introduction}

Myomectomy is a kind of surgery which reserves uterus. It not only reserves the reproductive function for young infertile women, but also maintains their lots of physiologic function, especially the internal secretion which can care women's physical and mental health. With the improvement of medical treatment technology, the development of peritoneoscope technology and the renovation of medical instruments, myomectomy has been developed from single abdominal type myomectomy with wound to the direction of minimally invasive

* Corresponding author.

E-mail address: Gaomh8686@163.com 
surgery.[1] Because laparoscopic surgery has such advantages as small incision and rapid restoration, this makes it has been accepted by many gynecologists and patients. Laparoscopic myomectomy has been applied widely. Now, the difficulty in laparoscopic myomectomy includes hemorrhagic volume and suture. Here, we have compared these two kinds of surgeries in accordance with clinic cases, and have concluded some valuable conclusions.

\section{Data and methods}

\subsection{Normal dada}

We have analyzed 301 cases about abdominal type myomectomy and 265 cases about laparoscopic myomectomy which were from January 2006 to December 2009 in our hospital. The patients' age in these cases is from 20 years old to 47 years old, and the average age is $36.17 \pm 5.26$. The size and number of hysteromyoma in these two groups of surgeries are different, which is shown in Table 1.

Table 1 Comparison between two groups of hysteromyoma's number and size

\begin{tabular}{|c|c|c|c|c|c|c|}
\hline & \multicolumn{2}{|c|}{$\begin{array}{c}\text { Number of hysteromyoma } \\
\text { (n) }\end{array}$} & \multicolumn{2}{|c|}{$\begin{array}{c}\text { Diameter of } \\
\text { hysteromyoma (n) }\end{array}$} & \multicolumn{2}{|c|}{$\begin{array}{c}\text { Position of hysteromyoma } \\
\text { (n) }\end{array}$} \\
\hline & Single & Multiple & $<5 \mathrm{~cm}$ & $>5 \mathrm{~cm}$ & $\begin{array}{l}\text { Under } \\
\text { serosa }\end{array}$ & $\begin{array}{c}\text { Between } \\
\text { Muscle wall }\end{array}$ \\
\hline Abdominal type & 163 & 138 & 145 & 156 & 34 & 267 \\
\hline Laparoscopic & 185 & 80 & 174 & 91 & 16 & 249 \\
\hline
\end{tabular}

\subsection{Methods}

Choice of cases: hysteromyoma can cause many diseases, such as bellyache, frequent micturition, sterility, pathological changes of uterine appendages and too much menses. And the medicine has no effects on it. We can use laparoscopic surgery to cure if the hysteromyoma's size is bigger than $6 \mathrm{~cm}$ or it grows rapidly or it has been caused too much menses or the medicine has no effect on it. On the other hand, we also can use the method if it belongs to subserous myoma or intramural myoma by means of type-B ultrasonic examination. Before the laparoscopic surgery, we should execute the test for cervical cytology, which can eliminate cervical carcinoma. And we also finish uterine apoxesis if the length of uterus is bigger than $15 \mathrm{~mm}$, which can eliminate the probability of endometrial malignant change. The patients' age should be in 40 years old. Otherwise, we should consider the patients' menses and cultivation. The youngest patient in this group is 20 years old, and the oldest is 47 years old. In those cases, only 39 cases patients hadn't been pregnant.[2]

- Anaesthesia: We use general anesthesia or continuous epidural anesthesia which combines balanced anesthesia and heart rate monitor.

- Abdominal type myomectomy: According to the traditional operation, we always cut the front wall hysteromyoma and the upper incision is vertical and the under incision is horizontal, which won't affect revascularization. The front wall hysteromyoma shouldn't be cut horizontal when we operate for infertile women. The bottom of uterus should be cut vertically. The back wall hysteromyoma can be cut directly. We'd better reduce the number of cut if the hysteromyoma is multiple. When we sew up the incision continuously from the bottom to top, and the last layer should like cesarean section which can make incision sleekly. 
- Laparoscopic myomectomy: Firstly, we cut a incision whose size is $10 \mathrm{~mm}$ under the umbilical ring. Then we inject into $\mathrm{CO} 2$. When the abdomen pressure reaches $12 \mathrm{mmHg}$, we put peritoneoscope in. On the left lower quadrant and right lower quadrant, we puncture a hole whose size is 5mmTrocar, and then put surgical instruments. We first check whether there is adherence in pelvic organs, and then check the number and size of hysteromyoma. After cutting hysteromyoma by using electrotome, we start to remove it. Another method is that we cut uterine serosa by using single-pole electric coagulation and the hysteromyoma will be exposed. Then we grip hysteromyoma and pull it by means of drill or forceps. So the hysteromyoma will be peeled. The surface of wound can be sutured interruptedly or continuously by means of figure-of-eight suture. If the diameter of hysteromyoma is bigger than $6 \mathrm{~cm}$, it is hard to expose it. We can use oxytocin to reduce uterus, then we can remove it. The active hemorrhage point on wound surface can be cured by means of double-pole electric coagulation. And the bleeding position can be stopped bleeding by means of figure-of-eight suture. The cut hysteromyoma can be taken out by means of electrical uterus morcellutor.[3]

- $\quad$ Processing by using statistics: We have used t-test, $\chi 2$-test (The difference is useful when $\mathrm{P}<0.05$.)

\section{Results}

\subsection{Comparison between these two groups of surgerie}

The two groups of surgeries' time hasn't any statistical significance ( $>0.05)$. And the hemorrhagic volume and hospital day differences between the two groups of surgeries have statistical significance $(\mathrm{P}<0.01)$. Table 2 shows the comparison between these two groups of surgeries.

Table 2 Comparison between these two groups of surgeries

\begin{tabular}{ccccc}
\hline & $\begin{array}{c}\text { Hemorrhagic } \\
\text { volume (ml) }\end{array}$ & $\begin{array}{c}\text { Operation } \\
\text { time (min) }\end{array}$ & $\begin{array}{c}\text { Time needed to return } \\
\text { to normal body } \\
\text { temperature (day) }\end{array}$ & $\begin{array}{c}\text { Hospital day } \\
\text { (day) }\end{array}$ \\
\hline $\begin{array}{c}\text { Abdominal type } \\
\text { myomectomy }\end{array}$ & $106.86 \pm 32.65$ & $87.18 \pm 36.80$ & $2.85 \pm 1.73$ & $7.79 \pm 2.10$ \\
$\begin{array}{c}\text { Laparoscopic } \\
\text { myomectomy }\end{array}$ & $85.23 \pm 30.37$ & $81.56 \pm 30.71$ & $1.41 \pm 1.05$ & $5.81 \pm 1.47$ \\
$\mathrm{t}$ & 8.1250 & 1.9574 & 11.7739 & 12.8284 \\
\hline $\mathrm{P}$ & $<0.01$ & $>0.05$ & $<0.01$ & $<0.01$ \\
\hline
\end{tabular}

\subsection{Postoperative complications}

Abdominal type myomectomy: the whole operation process is all right, and there isn't any postoperative complication. Laparoscopic myomectomy: there were two cases were replaced by abdominal type myomectomy because hemorrhagic volume was high. There wasn't any hurt in organs during the process of those two groups of surgeries.

\subsection{Postoperative procession}

All those two groups of patients should be accepted preventive antibiotic after operation. If someone's body temperature is higher than $38.5^{\circ} \mathrm{C}$, we can let them take Chinese medicine about activating blood circulation to dissipate blood stasis. To the patient with big uterus after myomectomy, we should let them take 
medroxyprogesterone acetate for two or three months. We also have suggested those patients not to be pregnant in one year.[4]

\section{Discussion}

\subsection{The advantages of laparoscopic myomectomy}

This kind of surgery has such advantages as small hurt, rapid restoration, short hospital days, no incision on abdomen, and so on. But it also has some restrictions such as it is not fit everybody. And the hospital days is very excellent by comparing with abdominal type myomectomy $(\mathrm{P}<0.01)$, which shows its advantages fully.

\subsection{Unsubstitutable significance of abdominal type myomectomy}

Although abdominal type myomectomy hasn't such advantages of hospital days and postoperative restoration, the traditional abdominal type myomectomy has wide range of application. And it can remove hysteromyoma with bigger average diameter. In this paper, we found that there were statistics differences in number and size of two groups of hysteromyoma $(\mathrm{P}<0.01)$ by comparing the general information. Patients with multi-hysteromyoma or big hysteromyoma can be cured by means of abdominal type myomectomy, which represent the unsubstitutable significance of abdominal type myomectomy.

\subsection{Characteristics of laparoscopic myomectomy}

Laparoscopic myomectomy is especially fits for the hysteromyoma where lies under perimetrium. And we are still looking for the curing methods about its operation skills, postoperative complications, and so on. In those 265 cases laparoscopic myomectomy of this paper, there are 91 cases whose average diameter are bigger than $5 \mathrm{~cm}(34.34 \%)$, and there are 3 cases whose average diameter are smaller than $5 \mathrm{~cm}(1.13 \%)$, which it is hard to operate and has been replaced by abdominal type myomectomy.[5] The key of success is that we must avoid more hemorrhagic volume which caused by big and deep incision during the process of operation.

\subsection{Characteristics of abdominal type myomectomy}

This kind of surgery is not resisted by patients' age, hysteromyoma's number, size and position. If the number of hysteromyoma is more than four, or the diameter is bigger than $5 \mathrm{~cm}$, or it locates between the surfaces of perimetrium, we can accept abdominal type myomectomy initially. To the hysteromyoma with big size and number, the key factor of success is controlling hemorrhagic volume and infection prevention.

Abdominal type myomectomy is a kind of surgery which can retain women's pregnant function. It is good for patients' health and childbirth if we use peritoneoscope, such as those women maybe accepts cesarean section later. But we should consider the following aspects:

- It is benefit for single hysteromyoma or its diameter is smaller than $5 \mathrm{~cm}$;

- We don't suggest patients to accept this kind of surgery if their hysteromyoma's diameter is bigger than $7 \mathrm{~cm}$, or they have multiple hysteromyoma, or the hysteromyoma lies at the bottom of uterus;

- Usually, it is hard to check the hysteromyoma by using peritoneoscope if it lies under the perimetrium. And this will cause the probability of recrudescence;

- It is not easy to take out the removed hysteromyoma. We can take out the removed hysteromyoma conveniently by means of comminuter;

- The doctor's suture technology must be very skillful. They'd better accept figure-of-eight suture or continuous suture or other methods. They must suture smartly in accordance with every patient's condition, which can stop bleeding. 


\section{5. Improvement of laparoscopic myomectomy}

The outstanding characteristic of laparoscopic myomectomy is that it cause little wound and side-affection, which can make body's inner environment be stable. Usually, the key step during the operation is reducing hemorrhagic volume, which can make operation be clear. It not only reduces the affection of abdominal cavity, but also the time of stopping bleeding. So the operation time can be reduced greatly. When we operate these surgeries, we first inject 20IU oxytocin into myometrium or inject $10 \%$ pituitrin 6IU into perimetrium which can reduce hemorrhagic volume. Then we cut the envelope of hysteromyoma and peel it by means of singlepole electric coagulation or ultrasound knife, which can reduce hemorrhagic volume. There are all kinds of methods which used to take out hysteromyoma. We mainly choose cylinder shell rotary cutter to cut hysteromyoma into pieces, and then take it out by means of a incision of 1.5 or $2.5 \mathrm{~cm}$. This can reduce operation time efficiently and widen the application of laparoscopic myomectomy.

If the patients don't want to retain their uterus, we can take hysterectomy. Otherwise, we should consider in accordance with accurate conditions. Firstly, the diameter of hysteromyoma should be less $8 \mathrm{~cm}$ or $10 \mathrm{~cm}$; Secondly, the number of hysteromyoma should be less two or three. And if the hysteromyoma lies under mucous membranes, we'd better use laparoscopic myomectomy. Whether laparoscopic myomectomy can be used in hysteromyoma which lies between intramurals hasn't been accepted widely. The opponents think that it is hard to take laparoscopic myomectomy in accordance with this condition, and it takes too much time, even it can be cleared entirely. In recent years, many scholars have concluded that laparoscopic mysomectomy has such advantages as long time, little hemorrhage and fast rehabilitation by comparing with abdominal type myomectomy. We think that laparoscopic myomectomy is asking too much of operators. So the operators should act according to their ability. In recent years, some medical practitioners have taken laparoscopic myomectomy by using symphysis pubis or posterior fornix as auxiliary means, which can increase mission success rate and safety.[6]

\subsection{Necessity of abdominal type myomectomy}

The main reasons of taking abdominal type myomectomy during the process of laparoscopic mysomectomy include serious pelvic adhesion and hemorrhagic volume. On the other hand, malignant change and bladder injury are also the reasons which turn to abdominal type myomectomy. There were three cases which needed to turn to abdominal type myomectomy during the process of laparoscopic mysomectomy: one case had history of cesarean section, and the front wall of uterus conglutinated urocyst omentum majus, which affected the surgery; In the other two cases, the hysteromyoma lied near uterus artery, so we turned to abdominal type myomectomy because of avoiding hemorrhoea. So whether laparoscopic mysomectomy can be accepted depends on the conditions of patients and the probable postoperative complications. On the other hand, the doctors' medical skills and instruments are also important. This kind of changing during the process of laparoscopic mysomectomy is not failed, and we should choose better surgery for patient, which can avoid the serious complications.

Those two kinds of operations have their own advantages respectively. The surgery doctor should know the hysteromyoma's number, size and position, so he can choose the best way. On the other hand, the surgery doctor must consider their ability if he wants to accept laparoscopic mysomectomy. Otherwise, the surgery maybe prolonged or too much hemorrhage, even cause serious complications. The skilled peritoneoscope technology is the precondition of laparoscopic myomectomy which is a kind of new technology. It has such advantages as short time, little hemorrhage and fast rehabilitation.[7] With the development of peritoneoscope technology, it must be the trend of myomectomy. 


\section{7. Significance of retaining uterus}

The function of retained ovary will be declined if uterus be cut. Some cases show that if uterus has been cut, whether the retained ovary number is one or two, the rate of ovary failure is $34 \%$, and it will be $54 \%$ after four years. So the menopausal syndrome, coronary disease and osteoporosis will be appeared ahead of time. The reasons which cause these diseases are:

- After the uterus having been cut, the ovary's blood-supply which from uterus will be cut, too. This can change ovary's blood circulation and increase ovary artery pressure, so the microcirculation will be damaged and lymphatic return will be obstructed, and this forms vicious circle eventually. So the follicle growth of ovary will be affected and declined and ovarian hormone will be reduced, which cause the functions of ovary decline.

- The ER and PR will be disappeared if uterus be cut, which disturbs receptor's operation period. And this will affect the function of ovary because receptor's operation period will not balance ovarian hormone.

- In physiological status, human body still has the reflecting way from uterine neck to pituitary. The sex gland hormone will be more by exciting uterine neck, so the function of ovary can be regulated. And the reflecting way will be disappear after cutting uterus. Obviously, young woman's uterus can't be cut rashly.

Hysterectomy will affect cardiovascular system. Hysterectomy will decline the function of ovary before menopause. And the exudation of estrogen will be reduced, which increases all kinds of arteriosclerotic lipoprotein density and reduces all kinds of resist arteriosclerotic lipoprotein density. Eventually, the bloodlipoids metabolism will be disordered and incidence rate of coronary disease will be increased. Uterus is not only the critical organ of hormone; it also produces lots of bioactivator and hormone, such as prostaglandin, relaxin and prolactin. And we have known that uterus can produce lots of prostaglandin I2(PGI2) and thromboxane A2(TXA2) which are all important local hormone which vary with sexual cycle. PGI2 is a kind of substance which can expand blood vessel strongly and depolymerize blood platelet, and it has the function which can prevent the formation of atherosclerosis before menopause. TXA2 is a kind of substance which can collect blood platelet strongly. The balance between these two kinds of substances is very important to sustain the function of coronary artery.

\subsection{Development and future of laparoscopic myomectomy}

Because laparoscopic myomectomy has been applied widely, and it has been developed with the introduction of modern technology. The instruments of laparoscopic myomectomy have been renewed and developed greatly. All kinds of instruments, such as gasless peritoneoscope, THL and so on, they all came from the purposes of little wound and low disturbance. On the other hand, the surgeons' skills have been improved greatly. With the development of computer technology, we can control the device flexibly and display the picture clearly by means of laparoscopic myomectomy with manipulator. With the development of electrics information technology, we can realize long-distance consultation, surgery and teaching by means of Internet. All these have shown that laparoscopic myomectomy has good prospects.

As minimally invasive surgery, laparoscopic myomectomy must be the first choice. With the development of science technology, laparoscopic myomectomy will be improved well and it has good prospects. But it can replace the traditional abdominal type myomectomy. It is only one of the choices. Every method must have their own advantages, and we should make a concrete analysis of concrete cases. Surgical doctor not only should grasp all kinds of surgery methods, but also form their own strong suit. We can't resolve all kinds of gynecilogical operation by only depending on one method. And we also can't laparoscopic myomectomy entirely. 


\section{Conclusions}

Laparoscopic myomectomy has been applied widely. With the development of technology, we can know that all kinds of bioactivator which produced by uterus. Uterus is one of the useful organ which can keep human have physical and mental health. So it can't be cut rashly. With the improvement of laparoscopic myomectomy operation skills and medical instruments, laparoscopic myomectomy has good prospects in the future.

\section{References}

[1] Tulandi T,Al-Took S. Endoscopic myomectomy-laparoscopy and hysteroscopy. Obstet Gynecol Clin North Am,1999,26:135

[2] Dubuisson JB. Gonadotrophin-releasing hormone agonist and myomectomy Clin Ther,1992,14:51

[3] Semm K, Mettler L. Technical progress in pelvic surgery via operation laparoscopy.Am J Obstet Gynecol,1980,138:121

[4] Lang jinghe. gynecology laparoscopic surgery of status, controversy and development. Chinese Journal of Obstetrics and Gynecology,1996,31(6):323

[5] Li guangyi. Practical gynecology laparoscopic surgery [M].Beijing: Peoples Medical Publishing House, 2006:408.

[6] Xue suhua, Huang wan,Fan xuemei. Analysis of Laparoscopic myomectomy 97 cases [J]. Chinese Maternal and Child Health,2003,18(9):544-545.

[7] Xu fengying. using tourniquet myomectomy analysis of 30 cases [J]. Shanghai Medical,2001,24(6):177178. 